\title{
Preface
}

\section{Embracing the Next Phase in Obstetric Anesthesiology}

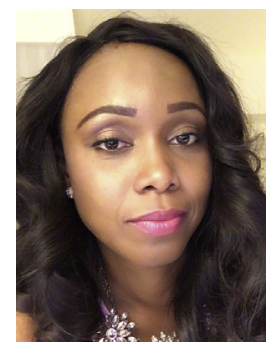

Onyi C. Onuoha, MD, MPH

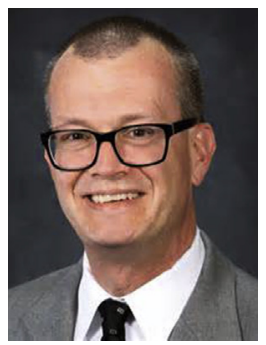

Robert R. Gaiser, MD

Editors

The accreditation of obstetric anesthesia by the Accreditation Council for Graduate Medical Education in the United States is the next phase in the evolution of the specialty. Prior to accreditation, the amount of scholarship, education, and research discovery in the field had been substantial with the practice addressing pertinent questions relating to the anesthetic implications in high-risk pregnancy; complications of pregnancy-related diseases; preterm labor; advances in labor analgesia; the use of noninvasive technology in labor and delivery; postcesarean analgesia; patient safety; fetal development; and advances in neonatology. With the ability to introduce structure and create national standards for fellows through the accreditation of the specialty, the next phase presents more opportunities for growth and innovation in research in the field of obstetric anesthesia.

Pioneering these efforts to educate the next generation are dedicated clinicians and researchers who continue to address challenging and pertinent questions by performing novel evidence-based research ranging from the primary care of the parturient to the use of noninvasive cutting-edge technology to improve the care of the parturient. With the changing demographics of the obstetric population, "the critically ill parturient," the field of obstetric anesthesia is continually evolving. The advancement of the specialty reflects the changing role of the obstetric anesthesiologist from a primarily procedural expert to a well-versed perioperative clinician collaborating with the nurse, obstetrician, neonatologist, and other consulting specialists in the multidisciplinary setting.

We are therefore thrilled to have such a talented group of experts contributing to this issue of Anesthesiology Clinics by addressing some of the relevant questions in obstetric anesthesia. This issue represents a comprehensive review, description, and update of current topics that add new knowledge to the field of obstetric anesthesia with the primary goal of improving maternal and fetal outcomes during pregnancy and the postpartum period. Additional insight is provided on controversial and debated topics like 
the link between neurocognitive outcomes and general anesthesia during the third trimester; the comparison between continuous epidural infusion and programmed intermittent epidural bolus for labor analgesia; the use of nitrous oxide in the laboring patient; obstetric versus anesthesia approaches to external cephalic version; and the current evidence on tilts and full stomach in patients undergoing cesarean delivery. Updates are included on ever-changing subject areas, such as the management of patients with preeclampsia, prophylaxis and management of obstetric hemorrhage, and the optimal pain management after cesarean delivery. And finally, topics delineating the future direction of obstetric anesthesia as in the use of ultrasound in obstetric anesthesia and huddles/debriefing to improve communication on labor and delivery are integrated in the content list. Ultimately, this issue highlights the various roles of the obstetric anesthesiologist in the care of the parturient.

We are grateful to all our contributors for their willingness to dedicate their time and effort writing this issue despite their busy schedules. We especially want to dedicate this issue to Dr Lee A. Fleisher, Chair of Anesthesia at the Hospital of the University of Pennsylvania and consulting editor, for his continuous support, mentorship, and investment in promoting education and research for resident and faculty development. Although not an obstetric anesthesiologist, his dedication to the growth of the specialty and scholarly pursuit as a whole inspires us and increases our drive to be more visibly well-rounded perioperative clinicians for the benefit and safety of our patients.

Onyi C. Onuoha, MD, MPH Department of Anesthesiology and Critical Care

Perelman School of Medicine University of Pennsylvania 3400 Spruce Street, Dulles 6 Philadelphia, PA 19104, USA

Robert R. Gaiser, MD

Department of Anesthesiology

University of Kentucky Lexington, KY 40506, USA 